\title{
Cocycles, Compatibility, and Poisson Brackets for Complex Fluids
}

\author{
Hernán Cendra*, Jerrold Marsden†, Tudor S. Ratiu ${ }^{\ddagger}$ \\ Dedicated to Alan Weinstein on the Occasion of his 60th Birthday \\ Vienna, August, 2003
}

\begin{abstract}
Motivated by Poisson structures for complex fluids containing cocycles, such as the Poisson structure for spin glasses given by Holm and Kupershmidt in 1988, we investigate a general construction of Poisson brackets with cocycles. Connections with the construction of compatible brackets found in the theory of integrable systems are also briefly discussed.
\end{abstract}

\section{Introduction}

Purpose of this Paper. The goal of this paper is to explore a general construction of Poisson brackets with cocycles and to link it to questions of compatibility similar to those encountered in the theory of integrable systems. The study is motivated by the specific brackets for spin glasses studied in Holm and Kupershmidt [1988].

In the body of the paper, we first describe several examples of Poisson brackets as reduced versions of, in principle, simpler brackets. Then we will show how this technique can be applied to study the compatibility of Poisson brackets. In the last section, we give the example of spin glasses and show that the Jacobi identity for the bracket given in Holm and Kupershmidt [1988] is a result of a cocycle identity.

Background. It is a remarkable fact that many important examples of Poisson brackets are reduced versions of canonical Poisson brackets. Perhaps the most basic case of this may be described as follows. Start with a configuration manifold $Q$ with

\footnotetext{
${ }^{1}$ Departamento de Matemática, Universidad Nacional del Sur, Av. Alem 1254, 8000 Bahia Blanca and CONICET, Argentina. uscendra@criba.edu.ar. Research partially supported by the EPFL.

${ }^{2}$ Control and Dynamical Systems, California Institute of Technology, Pasadena CA 91125. USA. marsden@cds.caltech.edu. Research partially supported by NSF grant DMS-0204474.

${ }^{3}$ Département de Mathématiques, École Polytechnique Fédérale de Lausanne. CH-1015 Lausanne. Switzerland. Tudor.Ratiu@epfl.ch. Research partially supported by the European Commission and the Swiss Federal Government through funding for the Research Training Network Mechanics and Symmetry in Europe (MASIE) as well as the Swiss National Science Foundation.
} 
a Lie group $G$ acting on $Q$ in such a way that $Q \rightarrow Q / G$ is a principal bundle (for instance, assume that the action is free and proper). Consider the cotangent space $T^{*} Q$ with its canonical symplectic and hence Poisson structure. As with general quotients of Poisson manifolds (see for instance Marsden and Ratiu [1999] for the relevant basic background material), the quotient space $\left(T^{*} Q\right) / G$ inherits a Poisson structure from $T^{*} Q$ in a natural way.

This construction of $\left(T^{*} Q\right) / G$ along with its quotient Poisson structure is a very important and rather general method for finding Poisson structures in mathematical physics, including fluid and plasma systems, and for obtaining brackets that satisfy the Jacobi identity by the nature of their construction (whereas verifying the Jacobi identity "by hand" could be quite arduous); see, for instance, Marsden and Weinstein [1982], Marsden and Weinstein [1983], Marsden, Ratiu, and Weinstein [1984a,b], Holm, Marsden, Ratiu, and Weinstein [1985], and Cendra, Holm, Hoyle, and Marsden [1998].

The detailed mathematical structure of the reduced Poisson brackets on quotients $\left(T^{*} Q\right) / G$ has itself a long and interesting history going back to the work of Sternberg [1977], Weinstein [1978], and Montgomery [1984], Montgomery [1986] on particles in Yang-Mills fields and the bundle picture in mechanics. Recent works in this area, such as Cendra, Marsden, and Ratiu [2001a] and Marsden and Perlmutter [2000] take the view that one should choose a connection on the shape space bundle $Q \rightarrow Q / G$ and then realize $\left(T^{*} Q\right) / G$ as the Whitney sum bundle $T^{*}(Q / G) \oplus \tilde{\mathfrak{g}}^{*}$, where $\mathfrak{g}$ is the Lie algebra of $G, \mathfrak{g}^{*}$ is its dual space, and where $\tilde{\mathfrak{g}}^{*}$ is the coadjoint bundle associated to the given $G$-action on $Q$ and the coadjoint action of $G$ on $\mathfrak{g}^{*}$. The reduced Hamilton equations on this space are called the Hamilton-Poincaré equations and are introduced and studied in Cendra, Marsden, Pekarsky and Ratiu [2003].

However, the construction of brackets on quotients of cotangent bundles is not the only case in which the idea of reduction is important to unify the study of otherwise mysterious Poisson brackets, some of which are well known from an algebraic point of view, namely that for spin glasses in Holm and Kupershmidt [1988]. A key feature of such brackets is the presence of cocycle terms.

Goals of this Paper. In this paper we shall present a fairly general construction of Poisson brackets with cocycles and show a simple reduction context for their study. We then show that the brackets of Holm and Kupershmidt [1988] may be viewed as examples of this construction. We will also make some suggestive links with compatible Poisson structures in the same sense as are found in the theory of integrable systems.

Future Directions. One of the key ideas that will be further developed in the future, especially in the context of complex fluids and similar examples, is to realize these cocycle terms as curvature (magnetic) terms associated with the reduction process itself. This point of view was explored in some detail in Marsden, Misiolek, Perlmutter, and Ratiu [1998] and Marsden, Misiolek, Ortega, Perlmutter, and Ratiu [2002] in the context of reduction by stages. The corresponding theory of Lagrangian 
reduction by stages was developed in Cendra, Marsden, and Ratiu [2001a] and it was used in Holm [2002] to study the variational approach to complex fluids. A similar reduction by stages method was used in Patrick [1999], who studied the Landau-Lifschitz equations, which occurs in magnetic materials.

It is our hope that our results will help provide a better understanding of not only spin glasses, but other important examples of complex fluids. The reader should realize that even the example of spin glasses has some obviously interesting additional and fundamental properties that require a deeper understanding, such as: when the spin glass bracket is "reduced" from a connection description to a "curvature" description, the cocycle disappears, as Holm and Kupershmidt [1988] point out. This seemingly miraculous property suggests that the overall reduction procedure is simply a Lie-Poisson reduction from the cotangent bundle of a group to the dual of its Lie algebra and that this one-step reduction may be realized as a two-stage reduction (the first by a normal subgroup), the cocycle is introduced at the first step of the reduction and then disappears in the second step, as it must since the final bracket is Lie-Poisson.

Another idea that requires further development is the connection of the global Poisson point of view presented here with the local, variational multisymplectic field theoretic point of view (see, for instance Gotay, Isenberg, and Marsden [1997], Marsden, Patrick, and Shkoller [1998] and Castrillón López, Ratiu, and Shkoller [2000]). In particular, it would be very interesting to explore the issue of cocycles and reduction by stages in the multisymplectic context and to link this theory to that of complex fluids. This is a relatively ambitious project that we do not address directly in this paper.

Parts of this paper are pedagogical in that we sometimes give two proofs of a result, sometimes by a direct coordinate computation; we feel that this may be beneficial for readers who want to see the same result from another viewpoint or who prefer an emphasis on coordinate calculations.

Preliminaries and Notation. Let $(M,\{\}$,$) be a Poisson manifold (see, for ex-$ ample, Marsden and Ratiu [1999] for an exposition and further references). It is well known that the Poisson structure $\{$,$\} , regarded as a skew symmetric bilin-$ ear mapping on $\mathcal{F}(M) \times \mathcal{F}(M)$, where $\mathcal{F}(M)$ denotes the space of smooth real valued functions on $M$, may alternatively be given in terms of a contravariant skewsymmetric two-tensor field $C \in \mathcal{T}_{0}^{2}(M)$ (the space of all two-tensors with indices up) on $M$. The relation between $\{$,$\} and C$ is given by the identity

$$
\{f, g\}=C(d f, d g)
$$

for any $f, g \in \mathcal{F}(M)$. Let us choose local coordinates $x^{i}$ on $M$, where $i=1, \ldots, m$, and $m$ is the dimension of $M$. Then the Poisson bracket may be written as follows

$$
\{f, g\}=C^{i j} \frac{\partial f}{\partial x^{i}} \frac{\partial g}{\partial x^{j}}
$$

where $C^{i j}$ are the components of $C$ in the chosen coordinate system and where the summation convention is in force. The Jacobi identity for the Poisson structure is, 
as is well-known, equivalent to the coordinate condition

$$
C^{i r} \frac{\partial C^{j k}}{\partial x^{r}}+C^{j r} \frac{\partial C^{k i}}{\partial x^{r}}+C^{k r} \frac{\partial C^{i j}}{\partial x^{r}}=0
$$

for all $i, j, k=1, \ldots, m$.

\section{A Bracket Associated to a 2-Form on a Symplectic Manifold}

Definition 2.1. Let $(P, \omega)$ be a symplectic manifold, and let $\Sigma$ be a given 2-form on $P$. For any given $f, g \in \mathcal{F}(P)$, the phase space $\Sigma$-bracket of $f$ and $g$ is defined by

$$
\{f, g\}_{\Sigma}=\Sigma\left(X_{f}, X_{g}\right)
$$

where $X_{f}$ and $X_{g}$ are the Hamiltonian vector fields associated to $f$ and $g$ respectively.

At this point we do not claim anything about the phase space $\Sigma$-bracket being a Poisson structure. We shall address this issue in due course.

Recall that the Lagrange bracket of two vector fields $X$ and $Y$ on a symplectic manifold $(P, \omega)$ is defined by the expression $[[X, Y]]:=\omega(X, Y)$ (see Abraham and Marsden [1978], page 196). Thus the phase space $\Sigma$-bracket of $f$ and $g$ may be viewed as a generalization of the Lagrange bracket of $X_{f}$ and $X_{g}$ to an arbitrary two-form on $P$. Note also that $\{f, g\}_{\omega}=\{f, g\}$ is the usual Poisson bracket associated to a symplectic manifold.

Let $P=T^{*} Q$ and denote by $\omega$ the canonical symplectic form on $P$. Let $\Sigma_{0}$ be a 2 -form on $Q$. If $\pi_{Q}: T^{*} Q \rightarrow Q$ is the canonical projection, $\Sigma=\pi_{Q}^{*} \Sigma_{0}$ is a 2 -form on $T^{*} Q$ and one can then form the corresponding phase space $\Sigma$-bracket using (2.1). In this cotangent bundle context, we will sometimes write $\Sigma_{0}$ for $\Sigma$ by a slight abuse of notation.

It is clear that the phase space $\Sigma$-bracket is skew-symmetric and is a derivation on each factor. The natural question is therefore to find the conditions on $\Sigma$ for $\{,\}_{\Sigma}$ to satisfy the Jacobi identity. We shall give below intrinsic as well as coordinate proofs of our assertions and shall study this problem in an increasing degree of generality.

The phase space $\Sigma$-bracket on the Cotangent Bundle of a Banach Space. We study the simplest case of a general construction by first considering the case of vector spaces. Let $V$ be a Banach space and $\Sigma_{0}: V \times V \rightarrow \mathbb{R}$ be a bilinear skew-symmetric map. The lift of this bilinear form to a bilinear form on $V \times V^{*}$ by means of the projection map $\pi_{V}: V \times V^{*} \rightarrow V$ is given by

$$
\Sigma\left(\left(v_{1}, \mu_{1}\right),\left(v_{2}, \mu_{2}\right)\right)=\Sigma_{0}\left(v_{1}, v_{2}\right)
$$

Consider the cotangent bundle $T^{*} V \equiv V \times V^{*}$. Note that the canonical projection $\pi_{V}: V \times V^{*} \rightarrow V$ is the projection on the first factor. To compute the phase space $\Sigma$-bracket, let $f \in \mathcal{F}\left(V \times V^{*}\right)$, the space of real valued functions on $V \times V^{*}$. 
Recall that the functional derivative of $f \in \mathcal{F}\left(V^{*}\right)$ at a point $\mu \in V^{*}$ is the element $\delta f / \delta \mu \in V$ defined by the identity

$$
\mathbf{D} f(\mu) \cdot \nu=\left\langle\nu, \frac{\delta f}{\delta \mu}\right\rangle, \quad \text { for all } \quad \mu, \nu \in V^{*},
$$

where $\mathbf{D} f(\mu)$ denotes the Fréchet derivative of $f: V^{*} \rightarrow \mathbb{R}$.

Since the Hamiltonian structure is canonical, $X_{f}(v, \mu)=(\delta f / \delta \mu,-\delta f / \delta v)$, and so we get

$$
\{f, g\}_{\Sigma}(v, \mu)=\Sigma\left(X_{f}, X_{g}\right)(v, \mu)=\Sigma_{0}\left(\frac{\delta f}{\delta \mu}, \frac{\delta g}{\delta \mu}\right)
$$

for all $v \in V$ and $\mu \in V^{*}$. This phase space $\Sigma$-bracket is actually a Poisson bracket as the next theorem shows.

Theorem 2.2. Let $V$ be a Banach space, $\Sigma_{0}: V \times V \rightarrow \mathbb{R}$ be a bilinear skewsymmetric map, and let $\Sigma$ be the induced bilinear skew symmetric form on $V \times V^{*}$ defined above. For given $f, g: V \times V^{*} \rightarrow \mathbb{R}$, the phase space $\Sigma$-bracket on $V \times V^{*}$

$$
\{f, g\}_{\Sigma}(v, \mu)=\Sigma_{0}\left(\frac{\delta f}{\delta \mu}, \frac{\delta g}{\delta \mu}\right), \quad \mu \in V^{*}
$$

satisfies the Jacobi identity.

A more general theorem will be proved in Theorem 2.6 below.

The Momentum $\Sigma$-bracket on the Dual of a Banach Space. Because the bracket (2.3) depends only on the variable $\mu$, one can also get a Poisson structure only on $V^{*}$ by a similar construction. It is obtained by Considering the functions $f$ and $g$ to be simply independent of the first factor.

Definition 2.3. Let $V$ be a Banach space and $\Sigma: V \times V \rightarrow \mathbb{R}$ be a bilinear skewsymmetric form. For given $f, g: V^{*} \rightarrow \mathbb{R}$, the momentum $\Sigma$-bracket on functions on $V^{*}$ is defined by

$$
\{f, g\}_{\Sigma}(\mu)=\Sigma\left(\frac{\delta f}{\delta \mu}, \frac{\delta g}{\delta \mu}\right), \quad \mu \in V^{*}
$$

We use the term "momentum $\Sigma$-bracket" since we think of elements of $V^{*}$ as momentum variables and the functions under consideration depend on $\mu \in V^{*}$ and not on $(v, \mu) \in T^{*} V$ as was the case in the preceding theorem. The momentum $\Sigma$-bracket is also a Poisson bracket, a result that is essentially equivalent to that in the preceding theorem.

Theorem 2.4. The momentum $\Sigma$-bracket given in Definition 2.3 satisfies the Jacobi identity and therefore defines a Poisson bracket on $V^{*}$.

We now give three proofs of Theorem 2.4, starting with a local one in finite dimensions. 
Calculus-based Proof. Assume that $V$ is finite dimensional. An immediate corollary of (1.2) is that any skew-symmetric constant tensor field $C \in \mathcal{T}_{0}^{2}(V)$ on a vector space defines a Poisson bracket. In particular, the bracket given by (2.4) is a Poisson bracket.

The following second proof also works for infinite dimensional Banach spaces.

Direct proof. As usual, the only thing we need to worry about is the Jacobi identity. Denote by $\Sigma^{b}: V \rightarrow V^{*}$ the map given by $\left\langle\Sigma^{b}(\xi), \eta\right\rangle=\Sigma(\xi, \eta)$, for any $\xi, \eta \in V$, where $\langle\rangle:, V^{*} \times V \rightarrow \mathbb{R}$ denotes the pairing between the vector space $V$ and its dual $V^{*}$. The following formula, obtained by differentiating (2.2) relative to $\mu$, will be used in the computation below:

$$
\left\langle\rho, \mathbf{D}\left(\mu \mapsto \frac{\delta f}{\delta \mu}\right)(\mu) \cdot \nu\right\rangle=\left\langle\nu, \mathbf{D}\left(\mu \mapsto \frac{\delta f}{\delta \mu}\right)(\mu) \cdot \rho\right\rangle=\mathbf{D}^{2} f(\mu)(\nu, \rho),
$$

for all $\mu, \nu, \rho \in V^{*}$. Thus, one has

$$
\frac{\delta}{\delta \mu}\{f, g\}_{\Sigma}(\mu)=-\mathbf{D}^{2} f(\mu)\left(\Sigma^{b}\left(\frac{\delta g}{\delta \mu}\right), \cdot\right)+\mathbf{D}^{2} g(\mu)\left(\Sigma^{b}\left(\frac{\delta f}{\delta \mu}\right), \cdot\right)
$$

so that for any $f, g, h: V^{*} \rightarrow \mathbb{R}$, it follows that

$$
\left\{\{f, g\}_{\Sigma}, h\right\}_{\Sigma}(\mu)=\mathbf{D}^{2} f(\mu)\left(\Sigma^{b}\left(\frac{\delta g}{\delta \mu}\right), \Sigma^{b}\left(\frac{\delta h}{\delta \mu}\right)\right)-\mathbf{D}^{2} g(\mu)\left(\Sigma^{b}\left(\frac{\delta f}{\delta \mu}\right), \Sigma^{b}\left(\frac{\delta h}{\delta \mu}\right)\right) .
$$

It readily follows that if one adds the other two terms obtained by circular permutations and uses the symmetry of the second derivative, the sum will vanish. This proves that $\{,\}_{\Sigma}$ is a Poisson bracket on $V^{*}$.

The third proof is by reduction. It may seem the most complicated at first, but in fact, it is the one that gives the most insight into generalizations of the result.

Proof by Reduction. Start with the cotangent bundle $T^{*} V=V \times V^{*}$. Let $V$ act on $V \times V^{*}$ as follows (cotangent lift of translations together with a momentum shift):

$$
\eta \cdot(\xi, \mu)=\left(\xi+\eta, \mu+\frac{1}{2} \Sigma^{b}(\eta)\right) .
$$

One checks that this action is Poisson with respect to the canonical cotangent bracket

$$
\{f, g\}_{\mathrm{can}}(\xi, \mu)=\left\langle\frac{\delta f}{\delta \xi}, \frac{\delta g}{\delta \mu}\right\rangle-\left\langle\frac{\delta g}{\delta \xi}, \frac{\delta f}{\delta \mu}\right\rangle .
$$

Now we simply Poisson reduce. The quotient space $\left(V \times V^{*}\right) / V$ is isomorphic to $V^{*}$ by the quotient map

$$
\pi:(\xi, \mu) \mapsto \mu-\frac{1}{2} \Sigma^{b}(\xi) .
$$

Since for $f: V^{*} \rightarrow \mathbb{R}$ we have

$$
\frac{\delta\left(\pi^{*} f\right)}{\delta \xi}=\frac{1}{2} \Sigma^{b}\left(\frac{\delta f}{\delta\left(\mu-\frac{1}{2} \Sigma^{b}(\xi)\right)}\right) \quad \text { and } \quad \frac{\delta\left(\pi^{*} f\right)}{\delta \mu}=\frac{\delta f}{\delta\left(\mu-\frac{1}{2} \Sigma^{b}(\xi)\right)},
$$


it follows that

$$
\begin{aligned}
\left\{\pi^{*} f, \pi^{*} g\right\}_{\text {can }}(\xi, \mu) & =\left\langle\frac{\delta\left(\pi^{*} f\right)}{\delta \xi}, \frac{\delta\left(\pi^{*} g\right)}{\delta \mu}\right\rangle-\left\langle\frac{\delta\left(\pi^{*} g\right)}{\delta \xi}, \frac{\delta\left(\pi^{*} f\right)}{\delta \mu}\right\rangle \\
& =\Sigma\left(\frac{\delta f}{\delta\left(\mu-\frac{1}{2} \Sigma^{b}(\xi)\right)}, \frac{\delta g}{\delta\left(\mu-\frac{1}{2} \Sigma^{b}(\xi)\right)}\right) \\
& =\{f, g\}_{\Sigma}\left(\mu-\frac{1}{2} \Sigma^{b}(\xi)\right) \\
& =\left(\pi^{*}\{f, g\}_{\Sigma}\right)(\xi, \mu) .
\end{aligned}
$$

Therefore, $\{f, g\}_{\Sigma}$ is the reduced bracket and hence is a Poisson bracket on $V^{*}$.

The $\Sigma$-Bracket on $T^{*} Q$. Let $Q$ be any given manifold and let $\Sigma_{0}$ be a 2-form on $Q$. Let $\pi_{Q}: T^{*} Q \rightarrow Q$ be the natural projection. Then $\Sigma=\pi_{Q}^{*} \Sigma_{0}$ is a 2 -form on $T^{*} Q$ and thus it has a phase space $\Sigma$-bracket associated to it, as explained before.

For any given 2 -form $\Sigma_{0}$ on $Q$ one has a well defined map

$$
\pi^{\Sigma_{0}}: T^{*} T Q \rightarrow T^{*} Q
$$

given in local coordinates by

$$
\pi^{\Sigma_{0}}\left(q^{i}, \delta q^{i}, p_{i}, \delta p_{i}\right):=\left(q^{i}, \delta p_{i}-\frac{1}{2} \Sigma_{0}(q)_{j i} \delta q^{j}\right)
$$

The map $\pi^{\Sigma_{0}}$ is defined at an element $w \in T_{v_{q}}^{*} T Q$ and paired with an element $u_{q} \in T_{q} Q$, by

$$
\left\langle\pi^{\Sigma_{0}}(w), u_{q}\right\rangle=\left\langle\left(\tau_{T^{*} Q} \circ \kappa_{Q}^{-1}\right)(w), u_{q}\right\rangle-\frac{1}{2} \Sigma_{0}\left(v_{q}, u_{q}\right),
$$

where $v_{q} \in T_{q} Q$. Here, $\tau_{T^{*} Q}: T T^{*} Q \rightarrow T^{*} Q$ denotes the tangent bundle projection and $\kappa_{Q}: T T^{*} Q \rightarrow T^{*} T Q$ is the canonical isomorphism. These maps are related to some constructions given by Tulczyjew [1977], but for expository clarity, we shall recall the details here. ${ }^{1}$

Intrinsic Characterization of the Map $\kappa_{Q}$. First of all, choose a local trivialization of $Q$, in which $Q$ is represented as an open set $U$ in a Banach space $E$. With such a choice, $T T^{*} Q$ is represented by $\left(U \times E^{*}\right) \times\left(E \times E^{*}\right)$, while $T^{*} T Q$ is represented by $(U \times E) \times\left(E^{*} \times E^{*}\right)$. In this representation, the map $\kappa_{Q}$ will turn out to be given by

$$
(q, p, \delta q, \delta p) \mapsto(q, \delta q, \delta p, p)
$$

We will show that the map $\kappa_{Q}$ is the unique map that intertwines two sets of maps. These maps are given as follows:

\footnotetext{
${ }^{1}$ We thank Alan Weinstein for some helpful correspondence on the geometry of iterated cotangent-tangent bundles.
} 
1. First Set of Maps. Consider the following two maps:

$$
\begin{aligned}
& T \pi_{Q}: T T^{*} Q \rightarrow T Q \\
& \pi_{T Q}: T^{*} T Q \rightarrow T Q
\end{aligned}
$$

which are the obvious maps (recall that we write $\pi_{R}: T^{*} R \rightarrow R$ for the cotangent projection). The first commutation condition that will be used to define $\kappa_{Q}$ is that

$$
\pi_{T Q} \circ \kappa_{Q}=T \pi_{Q}
$$

Thus, the diagram in Figure 2.1 should commute.

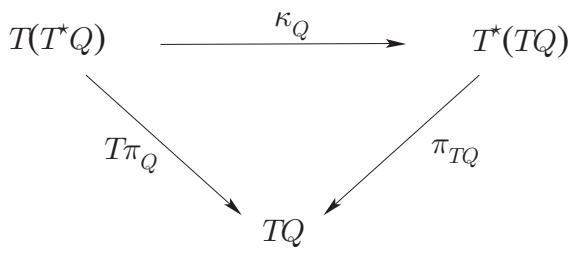

Figure 2.1: Commutative diagram for the cotangent analog of the canonical involution.

2. Second Set of Maps. The second set of maps is the following:

$$
\begin{aligned}
& \tau_{T^{*} Q}: T T^{*} Q \rightarrow T^{*} Q \\
& \pi^{0}: T^{*} T Q \rightarrow T^{*} Q .
\end{aligned}
$$

Recall that the tangent bundle projection of $T R$ to $R$ is denoted $\tau_{R}: T R \rightarrow R$. We need to explain the map $\pi^{0}$; let $\alpha_{v_{q}} \in T_{v_{q}}^{*} T Q$ and let $u_{q} \in T_{q} Q$. Then

$$
\left\langle\pi^{0}\left(\alpha_{v_{q}}\right), u_{q}\right\rangle=\left\langle\alpha_{v_{q}}, \operatorname{ver}\left(u_{q}, v_{q}\right)\right\rangle
$$

where

$$
\operatorname{ver}\left(u_{q}, v_{q}\right)=\left.\frac{d}{d t}\right|_{t=0}\left(v_{q}+t u_{q}\right) \in T_{v_{q}} T Q
$$

denotes the vertical lift of $u_{q}$ along $v_{q}$.

The second commutation condition is that

$$
\pi^{0} \circ \kappa_{Q}=\tau_{T * Q} .
$$

In other words, the diagram shown below in Figure 2.2 commutes. by

In a natural local trivialization, these four maps are readily checked to be given

$$
\begin{aligned}
T \pi_{Q}(q, p, \delta q, \delta p) & =(q, \delta q) \\
\pi_{T Q}(q, \delta q, p, \delta p) & =(q, \delta q) \\
\tau_{T^{*} Q}(q, p, \delta q, \delta p) & =(q, p) \\
\pi^{0}(q, \delta q, p, \delta p) & =(q, \delta p),
\end{aligned}
$$




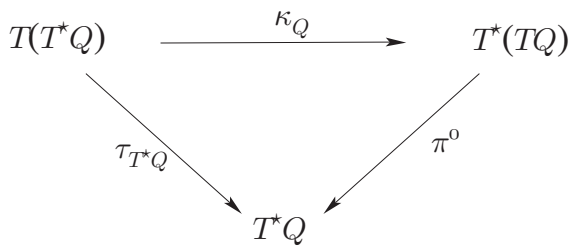

Figure 2.2: The second commutative diagram for the cotangent analog of the canonical involution.

from which it is easy to see that the commutation conditions are satisfied with the coordinate formula for $\kappa_{Q}$. It is clear that this uniquely characterizes the map $\kappa_{Q}$. We summarize what we have proved:

Proposition 2.5. For any manifold $Q$, there is a unique diffeomorphism

$$
\kappa_{Q}: T T^{*} Q \rightarrow T^{*} T Q
$$

such that the above two diagrams commute.

In summary, $\pi^{\Sigma_{0}}$ is defined by equation $(2.7)$, where $\kappa_{Q}$ is the map defined in the preceding proposition.

Cotangent Bundle $\Sigma$-bracket. Now we are ready to give the generalization of Theorem 2.2.

Theorem 2.6. Let $Q$ be a manifold and let $\Sigma_{0}$ be a 2-form on $Q$. Endow $T^{*} T Q$ with the canonical cotangent bundle symplectic structure. There is a unique Poisson structure on $T^{*} Q$ obtained by declaring $\pi^{\Sigma_{0}}: T^{*} T Q \rightarrow T^{*} Q$ to be a Poisson map; in fact, this bracket on $T^{*} Q$ is given by the cotangent $\Sigma_{0}$-bracket

$$
\{f, g\}_{\Sigma_{0}}(q, p)=\Sigma_{0}(q)\left(\frac{\delta f}{\delta p}, \frac{\delta g}{\delta p}\right)
$$

where $f, g \in \mathcal{F}\left(T^{*} Q\right)$ and where the fiber functional derivative $\delta f / \delta p \in T_{q} Q$ is defined by

$$
\left\langle\left(q, p^{\prime}\right), \frac{\delta f}{\delta p}\right\rangle=\left.\frac{d}{d t}\right|_{t=0} f\left(q, p+t p^{\prime}\right)
$$

for all $\left(q, p^{\prime}\right) \in T_{q}^{*} Q$.

We give two proofs. The first is a brute force coordinate proof and the second is by reduction.

Coordinate Proof. Let the two-form $\Sigma_{0}$ be written in components as

$$
\Sigma_{0}=\Sigma_{i j} d q^{i} \otimes d q^{j}
$$


where the sum is understood to be over all $i, j$ (no restriction on indices, such as $i<j$, which would introduce factors of 2) and where $\Sigma_{i j}$ is a function of $q$ alone. Then we have

$$
\{f, g\}_{\Sigma_{0}}=\Sigma_{i j} \frac{\partial f}{\partial p_{i}} \frac{\partial g}{\partial p_{j}} .
$$

The only axiom that is not obvious is the Jacobi identity. To check it, we compute directly in coordinates as follows:

$$
\begin{aligned}
\left\{f,\{g, h\}_{\Sigma_{0}}\right\}_{\Sigma_{0}} & =\Sigma_{k l} \frac{\partial f}{\partial p_{k}} \frac{\partial}{\partial p_{l}}\left(\Sigma_{i j} \frac{\partial g}{\partial p_{i}} \frac{\partial h}{\partial p_{j}}\right) \\
& =\Sigma_{k l} \Sigma_{i j} \frac{\partial f}{\partial p_{k}} \frac{\partial^{2} g}{\partial p_{l} \partial p_{i}} \frac{\partial h}{\partial p_{j}}+\Sigma_{k l} \Sigma_{i j} \frac{\partial f}{\partial p_{k}} \frac{\partial g}{\partial p_{i}} \frac{\partial^{2} h}{\partial p_{l} \partial p_{j}} .
\end{aligned}
$$

Therefore, we get

$$
\begin{aligned}
\left\{f,\{g, h\}_{\Sigma_{0}}\right\}_{\Sigma_{0}} & +\left\{g,\{h, f\}_{\Sigma_{0}}\right\}_{\Sigma_{0}}+\left\{h,\{f, g\}_{\Sigma_{0}}\right\}_{\Sigma_{0}} \\
& =\Sigma_{k l} \Sigma_{i j} \frac{\partial f}{\partial p_{k}} \frac{\partial^{2} g}{\partial p_{l} \partial p_{i}} \frac{\partial h}{\partial p_{j}}+\Sigma_{k l} \Sigma_{i j} \frac{\partial f}{\partial p_{k}} \frac{\partial g}{\partial p_{i}} \frac{\partial^{2} h}{\partial p_{l} \partial p_{j}} \\
& +\Sigma_{k l} \Sigma_{i j} \frac{\partial g}{\partial p_{k}} \frac{\partial^{2} h}{\partial p_{l} \partial p_{i}} \frac{\partial f}{\partial p_{j}}+\Sigma_{k l} \Sigma_{i j} \frac{\partial g}{\partial p_{k}} \frac{\partial h}{\partial p_{i}} \frac{\partial^{2} f}{\partial p_{l} \partial p_{j}} \\
& +\Sigma_{k l} \Sigma_{i j} \frac{\partial h}{\partial p_{k}} \frac{\partial^{2} f}{\partial p_{l} \partial p_{i}} \frac{\partial g}{\partial p_{j}}+\Sigma_{k l} \Sigma_{i j} \frac{\partial h}{\partial p_{k}} \frac{\partial f}{\partial p_{i}} \frac{\partial^{2} g}{\partial p_{l} \partial p_{j}} .
\end{aligned}
$$

The sum of the first and the last term is

$$
\begin{aligned}
& \Sigma_{k l} \Sigma_{i j} \frac{\partial f}{\partial p_{k}} \frac{\partial^{2} g}{\partial p_{l} \partial p_{i}} \frac{\partial h}{\partial p_{j}}+\Sigma_{k l} \Sigma_{i j} \frac{\partial f}{\partial p_{i}} \frac{\partial^{2} g}{\partial p_{l} \partial p_{j}} \frac{\partial h}{\partial p_{k}} \\
& =\Sigma_{k l} \Sigma_{i j} \frac{\partial f}{\partial p_{k}} \frac{\partial^{2} g}{\partial p_{l} \partial p_{i}} \frac{\partial h}{\partial p_{j}}+\Sigma_{j l} \Sigma_{k i} \frac{\partial f}{\partial p_{k}} \frac{\partial^{2} g}{\partial p_{l} \partial p_{i}} \frac{\partial h}{\partial p_{j}} \\
& =\left(\Sigma_{k l} \Sigma_{i j}+\Sigma_{j i} \Sigma_{k l}\right) \frac{\partial f}{\partial p_{k}} \frac{\partial^{2} g}{\partial p_{l} \partial p_{i}} \frac{\partial h}{\partial p_{j}},
\end{aligned}
$$

where in the first equality we renamed the summation indices and in the second we used symmetry of the mixed partial derivatives. This expression vanishes since $\Sigma_{i j}=-\Sigma_{j i}$. There are two similar pairs whose sum is also 0 by the same argument. Thus, Jacobi's identity holds.

Now we shall prove that $\pi^{\Sigma_{0}}:\left(T^{*} T Q,\{\},\right) \rightarrow\left(T^{*} Q,\{,\}_{\Sigma_{0}}\right)$ is a Poisson map. Let $f, g: T^{*} Q \rightarrow \mathbb{R}$ and denote by $\left(q^{i}, \delta q^{i}, p_{i}, \delta p_{i}\right)$ the canonical coordinates on $T^{*} T Q$. By definition of pull-back and the map $\pi^{\Sigma_{0}}$ we have

$$
\left(\pi^{\Sigma_{0}}\right)^{*} f\left(q^{i}, \delta q^{i}, p_{i}, \delta p_{i}\right)=f\left(q^{i}, \delta p_{i}-\frac{1}{2} \Sigma_{j i} \delta q^{j}\right)
$$


Therefore, by the chain rule, we have the following identities:

$$
\begin{aligned}
& \frac{\partial\left(\pi^{\Sigma_{0}}\right)^{*} f}{\partial q^{i}}=\frac{\partial f}{\partial q^{i}}-\frac{1}{2} \frac{\partial f}{\partial p_{j}} \frac{\partial \Sigma_{k j}}{\partial q^{i}} \delta q^{k} \\
& \frac{\partial\left(\pi^{\Sigma_{0}}\right)^{*} f}{\partial(\delta q)^{i}}=-\frac{1}{2} \Sigma_{i k} \frac{\partial f}{\partial p_{k}} \\
& \frac{\partial\left(\pi^{\Sigma_{0}}\right)^{*} f}{\partial p_{i}}=0 \\
& \frac{\partial f}{\partial(\delta p)_{i}}=\frac{\partial f}{\partial p_{i}}
\end{aligned}
$$

where the right hand sides are evaluated at the point $\left(q^{i}, \delta p_{i}-\frac{1}{2} \Sigma_{j i} \delta q^{j}\right)$. Therefore, the canonical Poisson bracket of $\left(\pi^{\Sigma_{0}}\right)^{*} f$ and $\left(\pi^{\Sigma_{0}}\right)^{*} g$ is given by

$$
\begin{aligned}
\left\{\left(\pi^{\Sigma_{0}}\right)^{*} f,\left(\pi^{\Sigma_{0}}\right)^{*} g\right\}= & \frac{\partial\left(\pi^{\Sigma_{0}}\right)^{*} f}{\partial q^{i}} \frac{\partial\left(\pi^{\Sigma_{0}}\right)^{*} g}{\partial p_{i}}+\frac{\partial\left(\pi^{\Sigma_{0}}\right)^{*} f}{\partial(\delta q)^{i}} \frac{\partial\left(\pi^{\Sigma_{0}}\right)^{*} g}{\partial(\delta p)_{i}} \\
& -\frac{\partial\left(\pi^{\Sigma_{0}}\right)^{*} g}{\partial q^{i}} \frac{\partial\left(\pi^{\Sigma_{0}}\right)^{*} f}{\partial p_{i}}-\frac{\partial\left(\pi^{\Sigma_{0}}\right)^{*} g}{\partial(\delta q)^{i}} \frac{\partial\left(\pi^{\Sigma_{0}}\right)^{*} f}{\partial(\delta p)_{i}} \\
= & -\frac{1}{2} \Sigma_{i k} \frac{\partial f}{\partial p_{k}} \frac{\partial g}{\partial p_{i}}+\frac{1}{2} \Sigma_{i k} \frac{\partial g}{\partial p_{k}} \frac{\partial f}{\partial p_{i}} \\
= & \Sigma_{i k} \frac{\partial g}{\partial p_{k}} \frac{\partial f}{\partial p_{i}}=\left(\pi^{\Sigma_{0}}\right)^{*}\{f, g\}_{\Sigma_{0}} .
\end{aligned}
$$

Uniqueness follows since $\left(\pi^{\Sigma_{0}}\right)^{*}$ is a surjective submersion.

Proof by Reduction. The strategy of the proof is to reduce the canonical Poisson structure on $T^{*} T Q$ to $T^{*} Q$ by means of the map $\pi^{\Sigma_{0}}$. Let us first recall some general facts about how one does such a procedure.

To motivate the construction, we note that if $\pi: P \rightarrow R$ is a submersive Poisson map from the Poisson manifold $P$ to the Poisson manifold $R$, and if $f, g \in \mathcal{F}(P)$ are such that for $z \in P, d f(z), d g(z)$ vanish on ker $T \pi(z)$, then $d\{f, g\}(z)$ vanishes on $\operatorname{ker} T \pi(z)$ as well. In fact, the condition on a one-form $\alpha \in T_{z}^{*} P$ needed for it to push down to a well defined one-form at $\pi(z) \in R$ is precisely that $\alpha$ vanish on the kernel of $T_{z} \pi$.

Now suppose that we want $R$ to inherit a Poisson structure from $P$. The condition needed for the Poisson structure to push down from $P$ to $R$ under a submersion $f: P \rightarrow R$ is exactly that if $f, g \in \mathcal{F}(P)$ are such that for $z \in P, d f(z), d g(z)$ vanish on $\operatorname{ker} T \pi(z)$, then $d\{f, g\}(z)$ vanishes on $\operatorname{ker} T \pi(z)$. If this condition holds, then it is a straightforward procedure to compute the reduced bracket on $R$, as in the last part of the preceding proof. This technique has been developed in a more general context by Marsden and Ratiu [1986] and has been used in interesting ways in the study of integrable systems (see, for instance Pedroni [1995]).

In our case, consider the foliation of $T^{*} T Q$ whose leaves are the level sets of the map $\pi^{\Sigma_{0}}$. To establish the theorem it is enough to show that for all functions 
$f, g: T^{*} T Q \rightarrow \mathbb{R}$, the condition $d f \mid \operatorname{ker} T \pi^{\Sigma_{0}}=0$ and $d g \mid \operatorname{ker} T \pi^{\Sigma_{0}}=0$ implies $d\{f, g\} \mid \operatorname{ker} T \pi^{\Sigma_{0}}=0$. Differentiating along a curve $\left(q^{i}(t), \delta q^{i}(t)\right)$, we find that

$$
\left.\frac{d}{d t}\right|_{t=0} \Sigma_{i j}(q) \delta q^{i}=\frac{\partial \Sigma_{i j}(q)}{\partial q^{k}} \dot{q}^{k} \delta q^{i}+\Sigma_{i j}(q) \dot{\delta q^{i}} .
$$

It follows that the tangent of the map $\pi^{\Sigma_{0}}$ is given by

$$
\begin{aligned}
& T \pi^{\Sigma_{0}}\left(q^{i}, \delta q^{i}, p_{i}, \delta p_{i}, \dot{q}, \dot{\delta q^{i}}, \dot{p}_{i}, \dot{\delta p_{i}}\right) \\
& \quad=\left(q^{j}, \delta p_{j}-\frac{1}{2} \Sigma_{i j}(q) \delta q^{i}, \dot{q}^{j}, \dot{\delta p_{j}}-\frac{1}{2}\left(\frac{\partial \Sigma_{i j}(q)}{\partial q^{k}} \dot{q}^{k} \delta q^{i}+\Sigma_{i j}(q) \dot{\delta q^{i}}\right)\right) .
\end{aligned}
$$

Thus, a typical tangent vector $\left(q^{i}, \delta q^{i}, p_{i}, \delta p_{i}, \dot{q}, \dot{\delta q^{i}}, \dot{p}_{i}, \dot{\delta p_{i}}\right)$ to the foliation can be written in the form

$$
\left(q^{i}, \delta q^{i}, p_{i}, \delta p_{i}, 0, \dot{\delta q^{i}}, \dot{p}_{i}, \frac{1}{2} \Sigma_{i j}(q) \dot{\delta q^{i}}\right)
$$

From this it follows that, for a given $f\left(q^{i}, \delta q^{i}, p_{i}, \delta p_{i}\right)$, the condition

$$
d f\left(q^{i}, \delta q^{i}, p_{i}, \delta p_{i}\right) \cdot\left(0, \dot{\delta q}^{i}, \dot{p}_{i}, \frac{1}{2} \Sigma_{i j}(q) \dot{\delta q^{i}}\right)=0
$$

is equivalent to

$$
\begin{aligned}
\frac{\partial f}{\partial p_{i}} & =0 \\
\frac{\partial f}{\partial \delta q^{i}}+\frac{1}{2} \frac{\partial f}{\partial \delta p_{j}} \Sigma_{i j}(q) & =0 .
\end{aligned}
$$

Assume that $f$ and $g$ satisfy (2.8) and (2.9). We must show that the canonical Poisson bracket on $T^{*} T Q$ given by

$$
\{f, g\}=\frac{\partial f}{\partial q^{i}} \frac{\partial g}{\partial p_{i}}+\frac{\partial f}{\partial \delta q^{i}} \frac{\partial g}{\partial \delta p_{i}}-\left(\frac{\partial g}{\partial q^{i}} \frac{\partial f}{\partial p_{i}}+\frac{\partial g}{\partial \delta q^{i}} \frac{\partial f}{\partial \delta p_{i}}\right)
$$

also satisfies (2.8) and (2.9). One can easily check that if both $f$ and $g$ satisfy (2.8) and (2.9), then

$$
\begin{aligned}
\{f, g\} & =\frac{1}{2} \Sigma_{i j}(q)\left(-\frac{\partial f}{\partial \delta p_{j}} \frac{\partial g}{\partial \delta p_{i}}\right)+\frac{1}{2} \Sigma_{i j}(q) \frac{\partial g}{\partial \delta p_{j}} \frac{\partial f}{\partial \delta p_{i}} \\
& =\Sigma_{i j}(q) \frac{\partial f}{\partial \delta p_{i}} \frac{\partial g}{\partial \delta p_{j}} .
\end{aligned}
$$

Using this expression it follows directly that (2.8) holds for $\{f, g\}$, that is,

$$
\frac{\partial\{f, g\}}{\partial p_{i}}=0
$$


It remains to show that (2.9) holds for $\{f, g\}$, that is,

$$
\frac{\partial}{\partial \delta q^{i}} \Sigma_{l k}(q) \frac{\partial f}{\partial \delta p_{l}} \frac{\partial g}{\partial \delta p_{k}}+\frac{1}{2} \Sigma_{i j}(q) \frac{\partial}{\partial \delta p_{j}} \Sigma_{l k}(q) \frac{\partial f}{\partial \delta p_{l}} \frac{\partial g}{\partial \delta p_{k}}=0
$$

Let us write, for short,

$$
\frac{\partial}{\partial \delta q^{i}} \Sigma_{l k}(q) \frac{\partial f}{\partial \delta p_{l}} \frac{\partial g}{\partial \delta p_{k}}=\alpha_{i}
$$

and

$$
\frac{1}{2} \Sigma_{i j}(q) \frac{\partial}{\partial \delta p_{j}} \Sigma_{l k}(q) \frac{\partial f}{\partial \delta p_{l}} \frac{\partial g}{\partial \delta p_{k}}=\beta_{i} .
$$

The $k$ th-components of $\alpha$ and $\beta$ are

$$
\begin{aligned}
\alpha_{k} & =\frac{\partial}{\partial \delta q^{k}} \Sigma_{i j}(q)\left(\frac{\partial f}{\partial \delta p_{i}} \frac{\partial g}{\partial \delta p_{j}}\right) \\
& =\Sigma_{i j} \frac{\partial^{2} f}{\partial \delta q^{k} \partial \delta p_{i}} \frac{\partial g}{\partial \delta p_{j}}+\Sigma_{i j} \frac{\partial f}{\partial \delta p_{i}} \frac{\partial^{2} g}{\partial \delta q^{k} \partial \delta p_{j}}
\end{aligned}
$$

and

$$
\begin{aligned}
\beta_{k} & =\frac{1}{2} \Sigma_{k l}(q)\left(\frac{\partial}{\partial \delta p_{l}}\left(\Sigma_{i j} \frac{\partial f}{\partial \delta p_{i}} \frac{\partial g}{\partial \delta p_{j}}\right)\right) \\
& =\frac{1}{2} \Sigma_{k l}(q)\left(\Sigma_{i j} \frac{\partial^{2} f}{\partial \delta p_{l} \partial \delta p_{i}} \frac{\partial g}{\partial \delta p_{j}}\right)+\frac{1}{2} \Sigma_{k l}(q)\left(\Sigma_{i j} \frac{\partial f}{\partial \delta p_{i}} \frac{\partial^{2} g}{\partial \delta p_{l} \partial \delta p_{j}}\right) .
\end{aligned}
$$

We have, by (2.9)

$$
\frac{\partial^{2} f}{\partial \delta p_{i} \partial \delta q^{k}}=\frac{1}{2} \Sigma_{l k} \frac{\partial^{2} f}{\partial \delta p_{i} \partial \delta p_{l}}
$$

and, similarly,

$$
\frac{\partial^{2} g}{\partial \delta p_{i} \partial \delta q^{k}}=\frac{1}{2} \Sigma_{l k} \frac{\partial^{2} g}{\partial \delta p_{i} \partial \delta p_{l}} .
$$

Replacing these expressions for $\partial^{2} f / \partial \delta p_{i} \partial \delta q^{k}$ and $\partial^{2} g / \partial \delta p_{i} \partial \delta q^{k}$ in the expression of $\alpha_{k}$ it follows that $\alpha_{k}+\beta_{k}=0$.

\section{Compatible Brackets}

Recall (see, for instance, Marsden and Weinstein $[1983]^{2}$ or Marsden and Ratiu [1999]) that if $\mathfrak{g}$ is a Lie algebra, its dual $\mathfrak{g}^{*}$ is a Poisson manifold relative to the Lie-Poisson bracket

$$
\{f, g\}(\mu)=\left\langle\mu,\left[\frac{\delta f}{\delta \mu}, \frac{\delta g}{\delta \mu}\right]\right\rangle,
$$

where $f, g: \mathfrak{g}^{*} \rightarrow \mathbb{R}, \mu \in \mathfrak{g}^{*}$, and $\langle\rangle:, \mathfrak{g}^{*} \times \mathfrak{g} \rightarrow \mathbb{R}$ denotes the canonical pairing between the Lie algebra and its dual. We now prove that the Lie-Poisson and the momentum $\Sigma$-bracket are compatible exactly when $\Sigma$ is a cocycle.

\footnotetext{
${ }^{2}$ Historical note: This is the paper that first proposed the name "Lie Poisson brackets", which is now in common use.
} 
Theorem 3.1. Let $\mathfrak{g}$ be a Lie algebra and $\Sigma: \mathfrak{g} \times \mathfrak{g} \rightarrow \mathbb{R}$ be a bilinear skew-symmetric map. For $f, g: \mathfrak{g}^{*} \rightarrow \mathbb{R}$, the Lie-Poisson bracket and the $\Sigma$-bracket are compatible, that is,

$$
\{f, g\}^{\Sigma}:=\{f, g\}+\{f, g\}_{\Sigma}
$$

is also a Poisson bracket on $\mathfrak{g}^{*}$, if and only if $\Sigma$ is a two-cocycle, that is, $\Sigma$ satisfies the identity

$$
\Sigma([\xi, \eta], \zeta)+\Sigma([\eta, \zeta], \xi)+\Sigma([\zeta, \xi], \eta)=0 \quad \text { for all } \quad \xi, \eta, \zeta \in \mathfrak{g} .
$$

\section{Remarks.}

1. As an important special case of a cocycle $\Sigma$, one can take an arbitrary coboundary, which is also known as the modified or constant Lie-Poisson bracket; that is, for each given $\nu \in \mathfrak{g}^{*}$ define

$$
\Sigma_{\nu}(\xi, \eta)=\langle\nu,[\xi, \eta]\rangle, \quad \xi, \eta \in \mathfrak{g}
$$

and consider the associated Poisson bracket $\{,\}^{\Sigma_{\nu}}$. This Poisson bracket appears in connection with several integrable systems and gives rise, via the associated recursion operator, to the commuting integrals of motion (see, for instance Ratiu [1980] for details).

2. In Libermann and Marle [1987], page 213 (chapter IV, Section 5.1, of the 1987 translation), there is a note on the sum of the canonical bracket on the dual of a Lie algebra plus a cocycle bracket which gives essentially the content of the preceding theorem. However, we give very direct proofs and, in addition, we give a more general version, not only because we do it in infinite dimensions, but also because of the more general Theorem 3.3 that follows of which Theorem 3.1 is a special case.

There are three interesting ways to prove the preceding theorem.

Coordinate Proof. Let $\left\{\xi_{1}, \ldots, \xi_{n}\right\}$ be a basis of the Lie algebra $\mathfrak{g}$ and denote by $C_{i j}^{k}$ the structure constants, that is, $C_{i j}^{k}$ are defined by

$$
\left[\xi_{i}, \xi_{j}\right]=C_{i j}^{k} \xi_{k}
$$

The Jacobi identity for the Lie bracket is equivalent to

$$
C_{i r}^{s} C_{j k}^{r}+C_{j r}^{s} C_{k i}^{r}+C_{k r}^{s} C_{i j}^{r}=0
$$

for all $i, j, k, s=1, \ldots, n$. The Lie-Poisson bracket has the coordinate expression

$$
\{f, g\}(\mu)=\left\langle\mu,\left[\frac{\delta f}{\delta \mu}, \frac{\delta g}{\delta \mu}\right]\right\rangle=\mu_{k} C_{i j}^{k} \frac{\partial f}{\partial \mu_{i}} \frac{\partial g}{\partial \mu_{j}},
$$

where $\mu=\mu_{i} \xi^{i}$ and $\left\{\xi^{1}, \ldots, \xi^{n}\right\}$ is the dual basis of $\left\{\xi_{1}, \ldots, \xi_{n}\right\}$ in $\mathfrak{g}^{*}$.

The momentum $\Sigma$-bracket has the coordinate expression

$$
\{f, g\}_{\Sigma}(\mu)=\Sigma_{i j} \frac{\partial f}{\partial \mu_{i}} \frac{\partial g}{\partial \mu_{j}}
$$


where, as before, $\Sigma=\Sigma_{i j} \xi^{i} \otimes \xi^{j}$ (with a sum over all $i, j$ ). Therefore, the coordinate expression of the sum bracket is

$$
\{f, g\}^{\Sigma}(\mu)=\left(\mu_{k} C_{i j}^{k}+\Sigma_{i j}\right) \frac{\partial f}{\partial \mu_{i}} \frac{\partial g}{\partial \mu_{j}} .
$$

Recall that (1.2) shows how to express the Jacobi identity on a Poisson manifold $(P,\{\}$,$) in terms of the components of the Poisson tensor. For our case, the quantity$ we must show vanishes for the validity of the Jacobi identity, is

$$
\begin{gathered}
\left(\mu_{s} C_{i r}^{s}+\Sigma_{i r}\right) \frac{\partial}{\partial \mu_{r}}\left(\mu_{s} C_{j k}^{s}+\Sigma_{j k}\right)+\left(\mu_{s} C_{j r}^{s}+\Sigma_{j r}\right) \frac{\partial}{\partial \mu_{r}}\left(\mu_{s} C_{k i}^{s}+\Sigma_{k i}\right) \\
\quad+\left(\mu_{s} C_{k r}^{s}+\Sigma_{k r}\right) \frac{\partial}{\partial \mu_{r}}\left(\mu_{s} C_{i j}^{s}+\Sigma_{i j}\right) \\
=\left(\mu_{s} C_{i r}^{s}+\Sigma_{i r}\right) C_{j k}^{r}+\left(\mu_{s} C_{j r}^{s}+\Sigma_{j r}\right) C_{k i}^{r}+\left(\mu_{s} C_{k r}^{s}+\Sigma_{k r}\right) C_{i j}^{r} \\
=\mu_{s}\left(C_{i r}^{s} C_{j k}^{r}+C_{j r}^{s} C_{k i}^{r}+C_{k r}^{s} C_{i j}^{r}\right)+\Sigma_{i r} C_{j k}^{r}+\Sigma_{j r} C_{k i}^{r}+\Sigma_{k r} C_{i j}^{r} .
\end{gathered}
$$

The term $\left(C_{i r}^{s} C_{j k}^{r}+C_{j r}^{s} C_{k i}^{r}+C_{k r}^{s} C_{i j}^{r}\right)$ vanishes by (3.3). The rest vanishes if and only if the cocycle identity (3.2) holds, since

$$
\Sigma(\eta,[\zeta, \theta])=\Sigma\left(\eta^{i} \xi_{i}, C_{j k}^{r} \zeta^{j} \theta^{k} \xi_{r}\right)=\Sigma_{i r} C_{j k}^{r} \eta^{i} \zeta^{j} \theta^{k},
$$

for $\eta, \zeta, \theta \in \mathfrak{g}$.

Direct Proof. To show that the two Poisson brackets are compatible we must establish Jacobi's identity, so we proceed to a direct computation of the term

$$
\left\{\{f, g\}^{\Sigma}, h\right\}^{\Sigma}=\{\{f, g\}, h\}+\left\{\{f, g\}_{\Sigma}, h\right\}+\{\{f, g\}, h\}_{\Sigma}+\left\{\{f, g\}_{\Sigma}, h\right\}_{\Sigma} .
$$

The sum of the first term and the other two obtained by circular permutations of $(f, g, h)$ vanishes because the Lie-Poisson bracket satisfies the Jacobi identity. The same is true for the last term because the $\Sigma$-bracket satisfies the Jacobi identity, as we have already proved. To compute the two cross terms we shall need the following formula which is readily checked:

$$
\frac{\delta}{\delta \mu}\{f, g\}(\mu)=\left[\frac{\delta f}{\delta \mu}, \frac{\delta g}{\delta \mu}\right]-\mathbf{D}^{2} f(\mu)\left(\operatorname{ad}_{\delta g / \delta \mu}^{*} \mu, \cdot\right)+\mathbf{D}^{2} g(\mu)\left(\operatorname{ad}_{\delta f / \delta \mu}^{*} \mu, \cdot\right) .
$$


Therefore, using (2.6) and (3.4) yields

$$
\begin{aligned}
\left\{\{f, g\}_{\Sigma}, h\right\}(\mu)+\{\{f, g\}, h\}_{\Sigma}(\mu) & \\
= & \left\langle\mu,\left[\frac{\delta}{\delta \mu}\{f, g\}_{\Sigma}, \frac{\delta h}{\delta \mu}\right]\right\rangle+\Sigma\left(\frac{\delta}{\delta \mu}\{f, g\}, \frac{\delta h}{\delta \mu}\right) \\
= & -\left\langle\operatorname{ad}_{\delta h / \delta \mu}^{*} \mu, \frac{\delta}{\delta \mu}\{f, g\}_{\Sigma}\right\rangle-\left\langle\Sigma^{b}\left(\frac{\delta h}{\delta \mu}\right), \frac{\delta}{\delta \mu}\{f, g\}\right\rangle \\
= & \mathbf{D}^{2} f(\mu)\left(\Sigma^{b}\left(\frac{\delta g}{\delta \mu}\right), \operatorname{ad}_{\delta h / \delta \mu}^{*} \mu\right)-\mathbf{D}^{2} g(\mu)\left(\Sigma^{b}\left(\frac{\delta f}{\delta \mu}\right), \operatorname{ad}_{\delta h / \delta \mu}^{*} \mu\right) \\
& \quad+\Sigma\left(\left[\frac{\delta f}{\delta \mu}, \frac{\delta g}{\delta \mu}\right], \frac{\delta h}{\delta \mu}\right) \\
& \quad+\mathbf{D}^{2} f(\mu)\left(\operatorname{ad}_{\delta g / \delta \mu}^{*} \mu, \Sigma^{b}\left(\frac{\delta h}{\delta \mu}\right)\right)-\mathbf{D}^{2} g(\mu)\left(\operatorname{ad}_{\delta f / \delta \mu}^{*} \mu, \Sigma^{b}\left(\frac{\delta h}{\delta \mu}\right)\right) .
\end{aligned}
$$

The sum of the four terms involving second derivatives and the terms obtained by circular permutations of $(f, g, h)$ vanishes in view of the symmetry of the second derivatives.

The sum of the third term and those obtained by circular permutations of $(f, g, h)$ vanishes if and only if the cocycle identity (3.2) holds.

Reduction Proof. This will be discussed after we prove the more general Theorem 3.3.

Affine Poisson Structures. The following calculations are similar to some of the previous coordinate calculations, but reveal that, instead of dealing with the dual of a Lie algebra, which is a Poisson manifold on a vector space in which the Poisson tensor depends linearly on $\mu \in \mathfrak{g}^{*}$, one can as well start with a slightly more general situation, in which the bracket depends affinely on $\mu$.

Let $(M,\{\}$,$) be a Poisson manifold and assume for the moment that M$ is a vector space. The Poisson structure $\{$,$\} defines a tensor field C \in \mathcal{T}_{0}^{2}(M)$ such that, for any given $f, g \in \mathcal{F}(M),(1.1)$ is satisfied. Let us choose linear coordinates $x^{i}$ on $M$, where $i=1, \ldots, m$ and $m$ is the dimension of $M$. Then the Poisson structure is written as follows

$$
\{f, g\}=C^{i j} \frac{\partial f}{\partial x^{i}} \frac{\partial g}{\partial x^{j}}
$$

where $C^{i j}$ are the components of $C$ in the chosen coordinate system. An immediate corollary of (1.2), which we have already utilized, is that for any skew-symmetric constant tensor $C \in \mathcal{T}_{0}^{2}(M)$ the structure on $M$ defined by (1.1) defines a Poisson bracket.

Now let us consider a skew-symmetric tensor field $C \in \mathcal{T}_{0}^{2}(M)$ depending linearly on $x$, that is,

$$
C=C_{s} x^{s}
$$


Then the Jacobi identity (1.2) becomes

$$
x^{s}\left(C_{s}^{i r} C_{r}^{j k}+C_{s}^{j r} C_{r}^{k i}+C_{s}^{k r} C_{r}^{i j}\right)=0
$$

By differentiation with respect to $x^{s}$ we obtain the equivalent condition

$$
C_{s}^{i r} C_{r}^{j k}+C_{s}^{j r} C_{r}^{k i}+C_{s}^{k r} C_{r}^{i j}=0,
$$

which, of course, coincides with (3.3). Let $a \in M$ be fixed and consider the constant Poisson structure $C(a)=C_{s} a^{s}$. Then consider the structure $C+C(a)$, whose components are $C^{i j}+C^{i j}(a)=\left(x^{s}+a^{s}\right) C_{s}^{i j}$. The Jacobi identity (1.2) becomes

$$
\left(x^{s}+a^{s}\right)\left(C_{s}^{i r} C_{r}^{j k}+C_{s}^{j r} C_{r}^{k i}+C_{s}^{k r} C_{r}^{i j}\right)=0
$$

which is satisfied because of (3.7). We have therefore proven that $C+C(a)$ is a Poisson structure that depends affinely on $x \in M$.

Example. Let $M=\mathfrak{g}^{*}$, where $\mathfrak{g}$ is a Lie algebra. Then, as we have remarked already above, the Lie-Poisson bracket is given by

$$
\{f, g\}=\left\langle\mu,\left[\frac{\delta f}{\delta \mu}, \frac{\delta g}{\delta \mu}\right]\right\rangle=\mu_{k} C_{i j}^{k} \frac{\partial f}{\partial \mu_{i}} \frac{\partial g}{\partial \mu_{j}},
$$

where $C_{i j}^{k}$ are the structure constants of the Lie algebra $\mathfrak{g}$, which depends linearly on $\mu$. From the previous results we can conclude that, for any $\mu_{0}$, the expression

$$
\{f, g\}=\left\langle\mu,\left[\frac{\delta f}{\delta \mu}, \frac{\delta g}{\delta \mu}\right]\right\rangle+\left\langle\mu_{0},\left[\frac{\delta f}{\delta \mu}, \frac{\delta g}{\delta \mu}\right]\right\rangle
$$

defines a Poisson bracket.

We must remark that any linear Poisson structure on a vector space, in the sense of (3.5), comes from a Lie algebra structure on the dual of the vector space.

This discussion proves the following theorem.

Theorem 3.2. If $C$ is a Poisson structure on the vector space $M$ that depends linearly on $x$ and if $a \in M$ is fixed then $C+C(a)$ is a Poisson structure.

Compatibility of brackets on $T^{*} Q$. Now we shall prove the following theorem, from which Theorem 3.1 can be deduced by reduction, as we will show in a moment.

Theorem 3.3. Let $Q$ be a manifold and $\Sigma_{0}$ a two-form on $Q$. The bracket

$$
\{,\}^{\Sigma_{0}}=\{,\}_{0}+\{,\}_{\Sigma_{0}}
$$

on $T^{*} Q$, where $\{,\}_{0}$ is the canonical Poisson bracket and where $\{,\}_{\Sigma_{0}}$ is the bracket defined in Theorem 2.6, satisfies the Jacobi identity if and only if $\Sigma_{0}$ is closed. 
Proof. By working in a canonical (Darboux) chart, we can assume without loss of generality that $Q$ is an open subset of $\mathbb{R}^{n}$. A point of $T^{*} Q$ is denoted in the usual way as $(q, p)=\left(q^{1}, \ldots, q^{n}, p_{1}, \ldots, p_{n}\right)$. However, in order to use the notation introduced in the previous paragraph, we shall denote $\left(q^{1}, \ldots, q^{n}, p_{1}, \ldots, p_{n}\right) \equiv\left(x^{1}, \ldots, x^{n}, \ldots, x^{2 n}\right)$.

The canonical Poisson structure on $T^{*} Q$ is given by the constant tensor field defined by $C_{0}^{i j}=-\delta^{i, j-n}$ if $j>n, C_{0}^{i j}=\delta^{i-n, j}$ if $i>n$, and $C_{0}^{i j}=0$ otherwise.

Now let $\Sigma_{0}$ be a two-form on $Q$. Then we have the bracket $\{F, K\}_{\Sigma_{0}}$ defined in Theorem 2.6, whose associated tensor field, using the notation $x^{i}$ instead of $q^{i}$ or $p_{i}$ for the coordinates of a point of $T^{*} Q$, is given by $C_{\Sigma}^{i j}=2 \Sigma_{i-n, j-n}$ if $i>n$ and $j>n$; $C_{\Sigma}^{i j}=0$, otherwise. Define the tensor field $C^{i j}=C_{0}^{i j}+C_{\Sigma}^{i j}$. It is antisymmetric because $C_{0}^{i j}$ and $C_{\Sigma}^{i j}$ are antisymmetric. Now let us see which condition $\Sigma_{0}$ must satisfy in order for $C^{i j}$ to be the associated tensor field to a Poisson bracket. This can be achieved using the coordinate expression of the Jacobi identity (1.2). Taking into account that $C_{\Sigma}^{i j}$ satisfies the Jacobi identity and the fact that the tensor field $C_{0}^{i j}$ is constant, it can be easily shown that the Jacobi identity reduces to

$$
C_{0}^{i r} \frac{\partial C_{\Sigma}^{j k}}{\partial x^{r}}+C_{0}^{j r} \frac{\partial C_{\Sigma}^{k i}}{\partial x^{r}}+C_{0}^{k r} \frac{\partial C_{\Sigma}^{i j}}{\partial x^{r}}=0
$$

from which, using the special form of $C_{0}^{i j}$, one can easily deduce the equivalent condition

$$
\frac{\partial \Sigma_{j k}}{\partial q^{i}}+\frac{\partial \Sigma_{k i}}{\partial q^{j}}+\frac{\partial \Sigma_{i j}}{\partial q^{k}}=0
$$

This last identity simply states that $\mathbf{d} \Sigma_{0}=0$, that is, $\Sigma_{0}$ is a closed two-form.

Proof of Theorem 3.1 by Reduction. If $Q \equiv G$ is a Lie group and $\Sigma_{0}$ is a leftinvariant closed two-form on $G$, then $\{,\}^{\Sigma_{0}}$ is a Poisson bracket on $T^{*} G$ if and only if $\Sigma_{0}$ is closed, which is equivalent to the statement that the restriction $\Sigma$ of $\Sigma_{0}$ to the Lie algebra $\mathfrak{g}$ of $G$ is a 2-cocycle. (This is easily seen by writing the standard formula for the three form $\mathbf{d} \Sigma_{0}$ acting on three left invariant vector fields as a sum of six terms. Three of these vanish by left invariance and the remaining terms are the terms that are involved in the cocycle identity). From this and the previous theorem we obtain easily a proof of Theorem 3.1, by reduction.

\section{Spin Glasses}

Holm and Kupershmidt [1988] show that the evolution equations for spin glasses is governed by an affine bracket on the dual of a Lie algebra. Formula (2.26a) of that article gives

$$
\partial_{t}\left(\begin{array}{c}
P_{i} \\
\rho \\
G_{\alpha} \\
A_{i}^{\alpha}
\end{array}\right)=-\left(\begin{array}{cccc}
P_{k} \partial_{i}+\partial_{k} P_{i} & \rho \partial_{i} & G_{\beta} \partial_{i} & \partial_{k} A_{i}^{\beta}-A_{k, i}^{\beta} \\
\partial_{k} \rho & 0 & 0 & 0 \\
\partial_{k} G_{\alpha} & 0 & t_{\alpha \beta}^{\gamma} G_{\gamma} & \delta_{\alpha}^{\beta} \partial_{k}+t_{\alpha \gamma}^{\beta} A_{k}^{\gamma} \\
A_{k}^{\alpha} \partial_{i}+A_{i, k}^{\alpha} & 0 & \delta_{\beta}^{\alpha} \partial_{i}+t_{\beta \gamma}^{\alpha} A_{i}^{\gamma} & 0
\end{array}\right)\left(\begin{array}{c}
\delta H / \delta P_{k} \\
\delta H / \delta \rho \\
\delta H / \delta G_{\beta} \\
\delta H / \delta A_{k}^{\beta}
\end{array}\right) .
$$


The Hamiltonian matrix in this equation is given by the Lie-Poisson structure on the dual of the Lie algebra $\mathfrak{X}(S)\left(\Lambda^{0} \oplus\left(\left(\Lambda^{0} \otimes \mathfrak{g}\right)(S)\left(\Lambda^{n-1} \otimes \mathfrak{g}^{*}\right)\right)\right)$ augmented by a two-cocycle. We shall show below how this bracket is obtained as a corollary of Theorem 3.1 and in the process introduce the dynamic variables and explain the above notation.

Consider two Lie algebras $\mathfrak{a}, \mathfrak{b}$ and a vector space $U$. Assume that $U$ is a representation space for $\mathfrak{a}$ and that $\mathfrak{a}$ acts on $\mathfrak{b}$, as a Lie algebra, i.e. there is a Lie algebra homomorphism $\mathfrak{a} \rightarrow \operatorname{Der}(\mathfrak{b})$, where Der $\mathfrak{b}$ denotes the derivations of the Lie algebra $\mathfrak{b}$. Consider $U \oplus \mathfrak{b}$ as a direct sum of Lie algebras, $U$ with the trivial Lie algebra structure. Then the diagonal action of $\mathfrak{a}$ on $U \oplus \mathfrak{b}$ is a Lie algebra action of $\mathfrak{a}$ on $U \oplus \mathfrak{b}$ and one can form the semidirect product Lie algebra $\mathfrak{a}(S)(U \oplus \mathfrak{b})$ whose bracket is given by

$$
\left[\left(\xi_{1}, u_{1}, \eta_{1}\right),\left(\xi_{2}, u_{2}, \eta_{2}\right)\right]=\left(\left[\xi_{1}, \xi_{2}\right], \xi_{1} \cdot u_{2}-\xi_{2} \cdot u_{1}, \xi_{1} \cdot \eta_{2}-\xi_{2} \cdot \eta_{1}+\left[\eta_{1}, \eta_{2}\right]\right),
$$

for $\xi_{i} \in \mathfrak{a}, \eta_{i} \in \mathfrak{b}$ and $u_{i} \in U$.

Now let us assume that $\mathfrak{b}=\mathfrak{c}$ (S) $W$, for $\mathfrak{c}$ a Lie algebra and $W$ a representation space of $\mathfrak{c}$. In addition, assume that $\mathfrak{a}$ acts on $\mathfrak{c}$ as a Lie algebra, that $W$ is also an $\mathfrak{a}$-module, and that these three actions are compatible in the sense that the following identity holds

$$
\xi \cdot(\sigma \cdot w)=(\xi \cdot \sigma) \cdot w+\sigma \cdot(\xi \cdot w)
$$

for $\xi \in \mathfrak{a}, \sigma \in \mathfrak{c}$ and $w \in W$. Then the diagonal action of $\mathfrak{a}$ on $\mathfrak{c}(S W$, given by $\xi \cdot(\sigma, w)=(\xi \cdot \sigma, \xi \cdot w), \xi \in \mathfrak{a}, \sigma \in \mathfrak{c}$, and $w \in W$, is a Lie algebra action of $\mathfrak{a}$ on $\mathfrak{c}$ (S) $W$. Then, taking in (4.1) $\mathfrak{b}=\mathfrak{c}(\mathrm{S} W$, we get

$$
\begin{aligned}
& {\left[\left(\xi_{1}, u_{1}, \sigma_{1}, w_{1}\right),\left(\xi_{1}, u_{1}, \sigma_{1}, w_{1}\right)\right]} \\
& =\left(\left[\xi_{1}, \xi_{2}\right], \xi_{1} \cdot u_{2}-\xi_{2} \cdot u_{1}, \xi_{1} \cdot \sigma_{2}-\xi_{2} \cdot \sigma_{1}+\left[\sigma_{1}, \sigma_{2}\right],\right. \\
& \left.\xi_{1} \cdot w_{2}-\xi_{2} \cdot w_{1}+\sigma_{1} \cdot w_{2}-\sigma_{2} \cdot w_{1}\right) \text {. }
\end{aligned}
$$

As a particular case, we shall take

- $\mathfrak{a}=\mathfrak{X}\left(\mathbb{R}^{n}\right)$, the Lie algebra of all vector fields on $\mathbb{R}^{n}$,

- $W=\Omega \otimes V$, where $\Omega$ is the space of all tensor fields on $\mathbb{R}^{n}$ of a given type and $V$ is a given vector space,

- $\mathfrak{c}=\Lambda^{0} \otimes \mathfrak{g}$, where $\Lambda^{0}$ denotes the space of smooth functions in $\mathbb{R}^{n}$ and $\mathfrak{g}$ is a given finite dimensional Lie algebra, with bracket

$$
\left[\left(f_{1} \otimes \xi_{1}\right),\left(f_{2} \otimes \xi_{2}\right)\right]=f_{1} f_{2} \otimes\left[\xi_{1}, \xi_{2}\right],
$$

for $f_{i} \in \Lambda^{0}$ and $\xi_{i} \in \mathfrak{g}, i=1,2$.

In addition, we assume that the $\mathfrak{a}$-representation on $W$ is given by

$$
X \cdot(\omega \otimes v)=£_{X} \omega \otimes v,
$$


for $X \in \mathfrak{X}\left(\mathbb{R}^{n}\right), \omega \in \Omega, v \in V$ and where $£$ denotes the Lie derivative. The Lie algebra action of $\mathfrak{a}$ on $\mathfrak{c}$ is given by

$$
X \cdot(f \otimes \xi)=£_{X} f \otimes \xi,
$$

for $X \in \mathfrak{X}\left(\mathbb{R}^{n}\right), f \in \Lambda^{0}, \xi \in \mathfrak{g}$. The $\mathfrak{c}$-representation on $W$ is given by

$$
(f \otimes \xi) \cdot(\omega \otimes v)=f \omega \otimes \xi \cdot v
$$

for $\xi \in \mathfrak{g}, f \in \Lambda^{0}, v \in V, \omega \in \Omega$. Using the derivation property of the Lie derivative, a direct verification shows that the compatibility identity (4.2), which in this case becomes

$$
X \cdot((f \otimes \xi) \cdot(\omega \otimes v))=(X \cdot(f \otimes \xi)) \cdot(\omega \otimes v)+(f \otimes \xi) \cdot(X \cdot(\omega \otimes v)),
$$

is satisfied. Therefore, we can form the semidirect product Lie algebra

$$
\mathfrak{X}\left(\mathbb{R}^{n}\right) \text { S }\left[\Lambda^{0} \oplus\left(\left(\Lambda^{0} \otimes \mathfrak{g}\right)(S(\Omega \otimes V))\right]\right.
$$

whose bracket is thus given by

$$
\begin{aligned}
& {\left[\left(X_{1}, g_{1}, f_{1} \otimes \xi_{1}, \omega_{1} \otimes v_{1}\right),\left(X_{2}, g_{2}, f_{2} \otimes \xi_{2}, \omega_{2} \otimes v_{2}\right)\right]} \\
& \quad=\left(\left[X_{1}, X_{2}\right], £_{X_{1}} g_{2}-£_{X_{2}} g_{1}, £_{X_{1}} f_{2} \otimes \xi_{2}-£_{X_{2}} f_{1} \otimes \xi_{1}+f_{1} f_{2} \otimes\left[\xi_{1}, \xi_{2}\right],\right. \\
& \left.\quad £_{X_{1}} \omega_{2} \otimes v_{2}-£_{X_{2}} \omega_{1} \otimes v_{1}+f_{1} \omega_{2} \otimes \xi_{1} \cdot v_{2}-f_{2} \omega_{1} \otimes \xi_{2} \cdot v_{1}\right) .
\end{aligned}
$$

This Lie algebra bracket coincides with the one given by formula (3.3) in Holm and Kupershmidt [1988].

If $V=\mathfrak{g}^{*}, \Omega=\Lambda^{n-1}\left(\mathbb{R}^{n}\right)$, the space of $(n-1)$-forms on $\mathbb{R}^{n}$, and the representation of $\mathfrak{g}$ on $\mathfrak{g}^{*}$ is the coadjoint representation $\xi \cdot \mu=-\operatorname{ad}_{\xi}^{*} \mu$, for $\xi \in \mathfrak{g}$ and $\mu \in \mathfrak{g}^{*}$, then the dual of the Lie algebra above is the phase space of spin glasses. Let us denote the variables in

$$
\left(\mathfrak{X}\left(\mathbb{R}^{n}\right) \text { S }\left[\Lambda^{0} \oplus\left(\left(\Lambda^{0} \oplus \mathfrak{g}\right) \mathbb{S}\left(\Lambda^{n-1} \otimes \mathfrak{g}^{*}\right)\right)\right]\right)^{*}=\Lambda^{1} \times \Lambda^{n} \times\left(\Lambda^{n} \otimes \mathfrak{g}^{*}\right) \times\left(\Lambda^{1} \otimes \mathfrak{g}\right)
$$

by $(P, \rho, G, A)$; here, $\Lambda^{k}$ denotes the space of $k$-forms on $\mathbb{R}^{n}$. In coordinates, $P_{i}$ is dual to $X^{i}, \rho$ is dual to $1 \in \Lambda^{0}, G_{\alpha}$ is dual to $1 \otimes e^{\alpha}$, where $e^{\alpha}$ is a basis of $\mathfrak{g}^{*}, A_{i}^{\alpha}$ is dual to $\left.\left(\partial_{i}\right\lrcorner d^{n} x\right) \otimes e^{\alpha} \in \Lambda^{n-1} \otimes \mathfrak{g}^{*}$.

Consider the skew symmetric bilinear map given by

$$
\begin{gathered}
\Sigma\left(\left(X_{1}, g_{1}, f_{1} \otimes \xi_{1}, \omega_{1} \otimes \mu_{1}\right),\left(X_{2}, g_{2}, f_{1} \otimes \xi_{2}, \omega_{2} \otimes \mu_{2}\right)\right) \\
=\int\left(d f_{1} \wedge \omega_{2}\right)\left\langle\mu_{2}, \xi_{1}\right\rangle-\left(d f_{2} \wedge \omega_{1}\right)\left\langle\mu_{1}, \xi_{2}\right\rangle .
\end{gathered}
$$

A direct verification, using the Stokes theorem and the usual exterior differential calculus, shows that $\Sigma$ satisfies the cocycle identity, which in this case comes down to proving that the sum of all cyclic permutations of

$$
\begin{gathered}
\Sigma\left(\left(\left[X_{1}, X_{2}\right], X_{1}\left[g_{2}\right]-X_{2}\left[g_{1}\right], X_{1}\left[f_{2}\right] \otimes \xi_{2}-X_{2}\left[f_{1}\right] \otimes \xi_{1}+f_{1} f_{2}\left[\xi_{1}, \xi_{2}\right],\right.\right. \\
\left.£_{X_{1}} \omega_{2} \otimes \mu_{2}-£_{X_{2}} \omega_{1} \otimes \mu_{1}+f_{1} \omega_{2} \otimes \xi_{1} \cdot \mu_{2}-f_{2} \omega_{1} \otimes \xi_{2} \cdot \mu_{1}\right), \\
\left.\left(X_{3}, g_{3}, f_{3} \otimes \xi_{3}, \omega_{3} \otimes \mu_{3}\right)\right)
\end{gathered}
$$


is zero. An alternative way to express (4.4) is the following

$$
\begin{gathered}
\Sigma\left(\left(X_{1}, g_{1}, f_{1} \otimes \xi_{1}, \omega_{1} \otimes \mu_{1}\right),\left(X_{2}, g_{2}, f_{2} \otimes \xi_{2}, \omega_{2} \otimes \mu_{2}\right)\right) \\
=\int\left(\left(\partial_{k} \omega_{1}^{k}\right) f_{2}\left\langle\mu_{1}, \xi_{2}\right\rangle+\left(\partial_{i} f_{1}\right) \omega_{2}^{i}\left\langle\mu_{2}, \xi_{1}\right\rangle\right) d^{n} x,
\end{gathered}
$$

which gives

$$
\Sigma^{b}(X, g, f \otimes \xi, \omega \otimes \mu)=\left(\begin{array}{cccc}
0 & 0 & 0 & 0 \\
0 & 0 & 0 & 0 \\
0 & 0 & 0 & \delta_{\alpha}^{\beta} \partial_{k} \\
0 & 0 & \delta_{\beta}^{\alpha} \partial_{i} & 0
\end{array}\right)\left(\begin{array}{c}
X^{i} \\
g \\
f \xi^{\beta} \\
\omega^{k} \mu_{\beta}
\end{array}\right)=\left(\begin{array}{c}
0 \\
0 \\
\partial_{k} \omega^{k} \mu_{\alpha} \\
\left(\partial_{i} f\right) \xi^{\alpha} .
\end{array}\right)
$$

Applying Theorem 3.1 it follows that the bracket given by the operator matrix

$$
-\left(\begin{array}{cccc}
P_{k} \partial_{i}+\partial_{k} P_{i} & \rho \partial_{i} & G_{\beta} \partial_{i} & \partial_{k} A_{i}^{\beta}-A_{k, i}^{\beta} \\
\partial_{k} \rho & 0 & 0 & 0 \\
\partial_{k} G_{\alpha} & 0 & t_{\alpha \beta}^{\gamma} G_{\gamma} & \delta_{\alpha}^{\beta} \partial_{k}+t_{\alpha \gamma}^{\beta} A_{k}^{\gamma} \\
A_{k}^{\alpha} \partial_{i}+A_{i, k}^{\alpha} & 0 & \delta_{\beta}^{\alpha} \partial_{i}+t_{\beta \gamma}^{\alpha} A_{i}^{\gamma} & 0
\end{array}\right)
$$

which coincides with the Hamiltonian matrix in formula (2.26a) of Holm and $\mathrm{Ku}-$ pershmidt [1988], defines a Poisson bracket.

Conclusions. In this paper we have given a glimpse at a possible connection of constructions of Poisson brackets with cocycles and brackets that one finds in the theory of complex fluids. We explicitly considered one example, namely that of spin glasses, following Holm and Kupershmidt [1988], but the approach hopefully is generalizable to other systems, such as those found in Holm [2002]. One of the longer term objectives of this endeavor would be to gain a deeper insight into the origins of cocycles. One approach to this is an algebraic one developed by Kuperschmidt [1985], but another, as mentioned earlier, is to view cocycles as magnetic terms that arise by reduction as the curvature of a connection. For example, the Bott-two cocycle can be viewed as arising this way and there is a rather general approach to this theory on both the Hamiltonian and Lagrangian sides, as shown in Marsden, Misiolek, Ortega, Perlmutter, and Ratiu [2002] and Cendra, Marsden, and Ratiu [2001a] respectively. We hope to pursue this line of thinking in the future as well as making links with multisymplectic geometry and reduction.

Acknowledgements. We are grateful to Larry Bates, Darryl Holm, and HansChristian Öttinger for helpful comments.

\section{References}

Abraham, R. and J. E. Marsden [1978], Foundations of Mechanics. BenjaminCummings Publ. Co, Updated 1985 version, reprinted by Perseus Publishing, second edition. 
Abraham, R., J. E. Marsden, and T. S. Ratiu [1988], Manifolds, Tensor Analysis and Applications, volume 75 of Applied Mathematical Sciences. Springer-Verlag, New York, second edition.

Arnold, V. I. [1989], Mathematical Methods of Classical Mechanics, volume 60 of Graduate Texts in Math. Springer-Verlag, First Edition 1978, Second Edition, 1989.

Castrillón López, M., T. S. Ratiu, and S. Shkoller [2000], Reduction in principal fiber bundles: Covariant Euler-Poincaré equations, Proc. Amer. Math. Soc. 128, $2155-2164$.

Cendra, H., D. D. Holm, M. J. W. Hoyle, and J. E. Marsden [1998], The MaxwellVlasov equations in Euler-Poincaré form, J. Math. Phys. 39, 3138-3157.

Cendra, H., J. E. Marsden, and T. S. Ratiu [2001a], Lagrangian Reduction by Stages, Memoirs of the American Mathematical Society, 152, Providence, R.I.

Cendra, H., J. E. Marsden, and T. S. Ratiu [2001b], Geometric mechanics, Lagrangian reduction and nonholonomic systems. In Enquist, B. and W. Schmid, editors, Mathematics Unlimited-2001 and Beyond, pages 221-273. Springer-Verlag, New York.

Cendra, H., J. E. Marsden, S. Pekarsky, and T. S. Ratiu [2003], Variational Principles for Lie-Poisson and Hamilton-Poincare Equations, Moscow Mathematical Journal (special issue for the 65th birthday of V. Arnold) (to appear).

Gotay, M., J. Isenberg, and J. E. Marsden [1997], Momentum Maps and the Hamiltonian Structure of Classical Relativistic Field Theories I, available at http://www.cds.caltech.edu/ marsden/.

Holm, D. D. [2002], Euler-Poincaré dynamics of perfect complex fluids, in Geometry, Mechanics and Dynamics, Ed . by P. Newton, P. Holmes, and A. Weinstein, Springer-Verlag, 113-168.

Holm, D. D. and B. A. Kupershmidt [1988], The analogy between spin glasses and Yang-Mills fluids, J. Math. Phys. 29, 21-30.

Holm, D. D., J. E. Marsden, T. S. Ratiu, and A. Weinstein [1985], Nonlinear stability of fluid and plasma equilibria, Phys. Rep. 123, 1-196.

Kupershmidt, B. A. [1985], Discrete Lax Equations and Differential-Difference Calculus, Astérisque, 212.

Libermann, P. and C. M. Marle [1987], Symplectic Geometry and Analytical Mechanics. Kluwer Academic Publishers.

Marsden, J., G. Misiolek, J. P. Ortega, M. Perlmutter, and T. Ratiu [2002], Symplectic Reduction by Stages, to appear. 
Marsden, J., G. Misiolek, M. Perlmutter, and T. Ratiu [1998], Symplectic reduction for semidirect products and central extensions, Diff. Geom. and its Appl. 9, 173212.

Marsden, J. E., G. W. Patrick, and S. Shkoller [1998], Multisymplectic geometry, variational integrators and nonlinear PDEs, Comm. Math. Phys. 199, 351-395.

Marsden, J. E. and M. Perlmutter [2000], The orbit bundle picture of cotangent bundle reduction, C. R. Math. Acad. Sci. Soc. R. Can. 22, 33-54.

Marsden, J. E. and T. Ratiu [1986], Reduction of Poisson manifolds, Lett. in Math. Phys. 11, 161-170.

Marsden, J. E. and T. S. Ratiu [1999], Introduction to Mechanics and Symmetry, volume 17 of Texts in Applied Mathematics, 1994, Second Edition, 1999, SpringerVerlag.

Marsden, J. E., T. S. Ratiu, and A. Weinstein [1984a], Semi-direct products and reduction in mechanics, Trans. Amer. Math. Soc. 281, 147-177.

Marsden, J. E., T. S. Ratiu, and A. Weinstein [1984b], Reduction and Hamiltonian structures on duals of semidirect product Lie Algebras, Contemp. Math. 28, 55100 .

Marsden, J. E. and A. Weinstein [1982], The Hamiltonian structure of the MaxwellVlasov equations, Physica D 4, 394-406.

Marsden, J. E. and A. Weinstein [1983], Coadjoint orbits, vortices and Clebsch variables for incompressible fluids, Physica D 7, 305-323.

Montgomery, R. [1984], Canonical formulations of a particle in a Yang-Mills field, Lett. Math. Phys. 8, 59-67.

Montgomery, R. [1986], The Bundle Picture in Mechanics, Ph.d. thesis, University of California Berkeley.

Öttinger, H. C. [2002], Modeling complex fluids with a tensor and a scalar as structural variables, Revista Mexicana de Fsica, 48, Supl. 1, 220-229. .

Patrick, G. W. [1999], The Landau-Lifshitz equation by semidirect product reduction, Lett. Math. Phys. 50, 177-188.

Pedroni, M. [1995], Equivalence of the Drinfeld-Sokolov reduction to a biHamiltonian reduction, Lett. Math. Phys. 35, 291-302.

Ratiu, T. S. [1980], Involution theorems. In Kaiser, G. and J. Marsden, editors, Geometric Methods in Mathematical Physics, volume 775 of Springer Lecture Notes, 219-257.

Sternberg, S. [1977], Minimal coupling and the symplectic mechanics of a classical particle in the presence of a Yang-Mills field, Proc. Nat. Acad. Sci. 74, 5253-5254. 
Tulczyjew, W. M. [1977], The Legendre transformation, Ann. Inst. Poincaré 27, $101-114$.

Weinstein, A. [1978], A universal phase space for particles in Yang-Mills fields, Lett. Math. Phys. 2, 417-420. 